\title{
The oculocardiac reflex as a surgical aid in identifying a slipped or 'lost' extraocular muscle
}

\author{
LEONARD APT AND SHERWIN J. ISENBERG \\ From the Department of Ophthalmology, Jules Stein Eye Institute, \\ UCLA School of Medicine, Los Angeles, California, USA
}

SUMMARY The oculocardiac reflex during strabismus surgery has generally been regarded as a hazard capable of causing death. Six cases are presented which show a beneficial use of the oculocardiac reflex. Isolation of a previously slipped or 'lost' extraocular muscle can be difficult. In this series identification of the tissue as muscle was substantiated by observing a positive oculocardiac reflex when traction was placed on the suspected tissue. Each of the 6 dislodged extraocular muscles was the medial rectus muscle. Three of the muscles had been resected and 3 either recessed or tenotomised. In one patient, despite 6 previous strabismus operations, including 2 strabotomies on a muscle that slipped, and in another patient, who had a lapse of 6 years since the last strabotomy, when the slipped muscle was isolated, the oculocardiac reflex could still be elicited. To avoid abolishing the oculocardiac reflex during surgery the anaesthetist should be instructed to avoid the use of an intravenous parasympatholytic agent, such as atropine, at the time of induction and during the operation.

Ophthalmologists and anaesthetists have regarded the oculocardiac reflex as an intraoperative hazard. This awareness is justified, since the resultant dysrhythmias have caused morbidity and even death. This reflex is usually caused by traction on the extraocular muscles, which, through the ophthalmic branch of the trigeminal nerve, stimulates the vagal centre. The vagus nerve then may cause a bradycardia or other dysrhythmia. A definition of a positive oculocardiac reflex has not been universally accepted. Some authors have used a bradycardia exceeding $10 \%$ of the premorbid heartbeat or any dysrhythmia as the criteria for a positive reflex. ${ }^{12}$ Because of our concern about this reflex we previously reviewed the topic and characterised the incidence of the reflex in regard to individual extraocular muscles, sex of patients, laterality, reproducibility, and type of general anaesthesia. ${ }^{3}$

The present report presents a beneficial use of the oculocardiac reflex. In the course of the previous study, and subsequently, we utilised the reproducibly positive oculocardiac reflex to identify a slipped or 'lost' muscle in a group of patients who had previous strabismus surgery.

The same anaesthetic routine and method of study was used in the group of patients with a slipped or 'lost' muscle as was used in the 243 patients in our previous report. Pethidine (Demerol)

Correspondence to Leonard Apt, MD, Jules Stein Eye Institute, UCLA School of Medicine, Los Angeles, California 90024 , USA. and atropine or scopolamine were given 1.5 to 2 hours before surgery. No atropine or scopolamine was given thereafter. Local anaesthesia was not used. Halothane, given endotracheally, was the general anaesthetic agent. When the patient was on the operating table, 4 electrocardiogram leads were attached to his extremities and a baseline recording of the cardiac rate and rhythm was made. If the rate and rhythm was normal, surgery began. The electrocardiogram ran continuously during the operation. After isolation of the tissue suspected to be the slipped or 'lost' muscle, traction was applied to it with a muscle hook or forceps. A positive oculocardiac reflex response was designated as any dysrythmia or a bradycardia of $10 \%$ or greater, as compared to the cardiac rate on the electrocardiogram tracing just before traction was applied.

We describe herein the clinical history and operative findings in 6 patients.

\section{Case reports}

CASE 1

An 11-year-old boy came to the Jules Stein Eye Institute with a history of congenital esotropia. He had undergone 6 previous operations on the extraocular muscles. At $\mathbf{3 0}$ months of age he had a recession of both medial rectus muscles, followed 1 year later by tenotomies of both inferior oblique muscles. Seven months later he had a myectomy of the right inferior oblique muscle and a resection of the left 
lateral rectus muscle. At 6 years of age a recession of the right superior rectus muscle and a resection of the right lateral rectus muscle was performed. At 9 years of age, to correct the residual esotropia, the surgeon re-recessed the left medial rectus muscle. Postoperatively the left eye was exotropic 20 prism dioptres and did not adduct past the midline. His sixth operation, performed 1 year later, consisted of lysis of adhesions around the supposed left medial rectus tendon and advancement of the supposed tendon. The left exotropia persisted postoperatively, and the patient was referred for consultation to one of the authors (L.A.).

Examination of the patient's eyes showed a left exotropia of 20 prism dioptres at distance and 30 prism dioptres at near, a left hypertropia of 6 prism dioptres, and an inability to adduct the left eye past the midline.

At operation forced ductions under general anaesthesia showed a moderate resistance to adduction of the left eye. At the original insertion site of the left medial rectus a band of tissue was found and a biopsy was done. The biopsy specimen later revealed fibrous connective tissue with inflammatory changes but no striated muscle fibres. Careful repeated passage of a muscle hook into the nasal area of the posterior globe yielded a small band of pink tissue. Before disinserting the band of pink tissue, to be sure the tissue contained extraocular muscle, traction was applied while monitoring the heart rate on an electrocardiogram. This manoeuvre caused the heart rate to decrease from 96 to 65 beats per minute. The band was disinserted and attached to the sclera $4 \mathrm{~mm}$ behind the limbus. In addition the left lateral rectus muscle was recessed $7 \mathrm{~mm}$, and adhesions around it were lysed. The left inferior oblique muscle, found attached to the original insertion site, was recessed $10 \mathrm{~mm}$. A stay suture was used to anchor the left eye in an adducted position for 6 days.

Postoperatively the left eye was mildly esotropic, but adduction of the left eye was regained.

\section{CASE 2}

A 26-year-old woman had a congenital left sixth nerve palsy. At 18 years of age her previous ophthalmologist performed an operation on the left eye, which consisted of transpositions of portions of the superior and inferior rectus muscles to the area of the lateral rectus muscle and a 'controlled tenotomy' of the medial rectus muscle. Postoperatively she developed an exotropia, mild dilatation of the left pupil, and head turn.

At 24 years of age she came to us with a visual acuity correctable to $6 / 6(20 / 20)$ in the right eye and $6 / 7 \cdot 5(20 / 25)$ in the left eye. She had a head turn to the right and a left exotropia of 32 prism dioptres at distance and 38 prism dioptres at near. Movement of the left eye was slight in abduction, and no movement occurred beyond the midline in adduction. Interestingly, the left palpebral fissure narrowed in adduction and widened in abduction.

At surgery, done under general anaesthesia, forced duction manoeuvres indicated resistance of the left eye to adduction and to elevation. The area of the left lateral rectus muscle was cleared of adhesions. During an attempt to isolate the left medial rectus muscle the hook encountered a fibrous-appearing band located only $2 \mathrm{~mm}$ behind the original insertion site. Decrease in the heart beat from 80 to 65 beats per minute was caused by traction on this band. The left lateral rectus muscle was recessed $8 \mathrm{~mm}$ and the newly identified left medial rectus muscle resected $7 \mathrm{~mm}$. Traction sutures kept the eye in adduction postoperatively.

In the $2 \frac{1}{2}$ years since the operation the patient has had no head turn nor any heterotropia in the primary position. Both abduction and adduction of the left eye have improved but remain limited.

\section{CASE 3}

This girl was 5 years old when she was first seen at the Jules Stein Eye Institute. At 3 years of age she had a recession of the right medial rectus and a tuck of the right lateral rectus muscle for esotropia. She developed an incomitant exotropia-40 prism dioptres in left gaze, 18 prism dioptres in primary position, and no deviation on right gaze. Six months later surgical exploration disclosed tissue, thought to be the right medial rectus, located $15 \mathrm{~mm}$ from the limbus. This tissue was advanced to the original medial rectus insertion site. The eye alignment did not change after the second operation.

Our examination showed 6/9 (20/30) visual acuity in each eye, 5 prism dioptres exophoria with left head turn, but with the head held straight, an exotropia of 25 prism dioptres at distance and 30 prism dioptres at near. The right eye was capable of only slight adduction past the midline.

At operation forced duction tests under general anaesthesia were negative. The sclera at the original insertion site of the right medial rectus was thin, with dark uvea shining through. Exploration revealed a band of tissue located about $13 \mathrm{~mm}$ from the limbus. Traction on this band decreased the heart rate from 110 to 70 beats per minute. The band, which appeared mostly fibrotic, was attached $4 \mathrm{~mm}$ from the limbus below the area of thin sclera. The right lateral rectus was recessed $6 \mathrm{~mm}$.

After the operation, the patient had no head turn, a small-angle esotropia, and good adduction of the right eye. 
CASE 4

A 5-year-old girl had intermittent exotropia since 9 months of age. She had a recession of the right lateral rectus and a resection of the right medial rectus muscles. On discharge from the hospital the morning after surgery the eyes were straight, but when the patient was seen on the sixth day after surgery she had a right exotropia of 30 prism dioptres with markedly decreased adduction of the right eye. The palpebral fissure widened in attempted adduction.

Eight days after the first operation she was operated upon at the Jules Stein Eye Institute. Forced duction tests under general anaesthesia were negative. At operation 1 double-armed and 1 single-armed suture, used to attach the right medial rectus muscle to the sclera, were still in position with the knots apparent. Only some strands of muscle were attached at the superior end of the insertion site. In exploring for the remainder of the muscle traction applied to the fibres which were thought to be muscle caused the heart rate to decrease from 100 to 85 beats per minute. These fibres were then attached to the original insertion site.

Three months after surgery the patient had an intermittent exotropia of 16 prism dioptres at distance and 22 prism dioptres at near with good adduction of the right eye.

\section{CASE 5}

This patient developed a large-angle exotropia in infancy. At 4 years of age her left lateral rectus muscle was recessed, and her left medial rectus muscle was resected. On the first postoperative day a small amount of esotropia was present. The left eye demonstrated normal ductions. Two days after surgery (and 1 day after discharge), while the child was thrashing and jumping about at home during a temper tantrum, her father noted a sudden 'turning out and no movement' of the left eye. On examination she had a left exotropia of 50 prism dioptres, inability to adduct the left eye past the midline, and widening of the palpebral fissure on attempted adduction.

Five days after her first operation she was returned to the operating room. Forced duction manoeuvres under general anaesthesia demonstrated a mild resistance to adduction of the left eye. Dissection at the original insertion site of the left medial rectus revealed that the double-armed suture was in place with a secure knot, but there was no tissue within the suture; only a bare scleral bed was visible. A muscle-like substance was found within Tenon's capsule. Traction on this tissue mass caused the heartbeat to decrease from 105 to 80 beats per minute. The tissue was advanced to the original insertion site, and reattached. Two stay sutures were used to keep the eye adducted during the immediate postoperative period.

The resulting eye alignment was slight left exotropia with a mild decrease in the ability of the left eye to adduct.

\section{CASE 6}

This girl was 6 years of age when the diagnosis was made of a left exotropia associated with a double elevator palsy of the left eye. Surgery performed on the left eye consisted of upward transposition and recession of the lateral rectus muscle and upward transposition and resection of the medial rectus muscle.

The postoperative course was uneventful until the fourth day, when the father noted the sudden onset of the 'left eye going out'. Trauma was denied. On examination the patient had a large left exotropia with no adduction of the left eye past the midline.

Surgery was performed 7 days after the initial operation. Under general anaesthesia the forced duction test was negative. In the area of the insertion of the left medial rectus muscle the suture and knot were found properly in place in the sclera. However, no muscle was held within the suture. Slightly behind the equator a structure which appeared to be muscle was found. Traction on the structure produced a decrease in heart rate from 104 to 88 beats per minute. The muscle was advanced and sutured to the sclera in the previously intended site.

After operation most of the action of the left medial rectus muscle returned.

\section{Discussion}

Diagnosis of a slipped or 'lost' extraocular muscle shortly after strabismus surgery is suspected when limitation of globe movement in the field of action of the dislodged muscle is associated with a marked overcorrection or undercorrection in the primary position, or a sudden large shift in ocular alignment from that noted immediately after surgery. Voluntary movement of the globe beyond the midline is usually minimal. On attempted movement of the eye in the field of action of the dislodged muscle the eye protrudes and the palpebral fissure widens, because the antagonist muscle relaxes while the disengaged agonist has no retraction effect on the globe.

We want to emphasise another diagnostic clinical sign derived from our series of dislodged medial rectus muscles, namely, the amount of exotropia at near exceeds the amount of exotropia at distance. In the case of a dislodged lateral rectus muscle one 
could expect the exotropia to be greater at distance than at near.

During the first weeks after a muscle is lost or has slipped the forced duction test is usually negative. However, after a few months the antagonist muscle may become tight, develop a contracture, and cause limited forced traction in the field of action of the disinserted muscle. At this stage enophthalmos may occur on gaze in the direction of reduced motility because the tight antagonist does not fully relax.

Saccadic velocity measurements in the early postoperative period reveal a reduced saccadic velocity of the posteriorly slipped muscle. A difference between agonist and antagonist saccadic velocity of $20 \%$ or greater strongly suggests a diagnosis of a slipped rectus muscle. ${ }^{4}$

The active force generation test described by $\mathrm{Scott}^{5}$ may disclose reduced or absent contracting power of the disengaged muscle. Scott ${ }^{6}$ also devised an electronic stimulator to identify a dislodged rectus muscle at the time of surgery.

Surgery for a suspected slipped or 'lost' rectus muscle is easier if performed soon after the muscle is dislodged, because the suture may still be seen attached to the muscle, and the muscle still has its reddish colour. Identification of the muscle may be more difficult, however, if a few weeks or months elapsed after the muscle disengaged. The area to be explored often has adhesions and extraneous tissue. As the surgeon seeks the muscle in question, he may have to lyse adhesions and judge if the tissue uncovered is or is not extraocular muscle. The muscle, especially the medial rectus, may have retracted into Tenon's capsule, and exploration along Tenon's capsule may reveal the muscle. Certain muscles have some form of weak attachment to other extraocular muscles that can aid the surgeon's exploration. For example, the lateral and inferior rectus muscles may be found by following the course of the inferior oblique muscle. Similarly, the superior rectus muscle may be found by following the course of the superior oblique tendon. ${ }^{7}$ Because the medial rectus muscle lacks even a loose attachment to another muscle, it is the muscle that usually is the most difficult to retrieve. In our 6 cases the medial rectus was the muscle that became dislodged.

A general belief is that a resected muscle tends to slip more than a recessed muscle, because of the greater tension placed on it. This may be true, but only half of the lost medial rectus muscles in our series had been resected, and the other 3 were either recessed or tenotomised.

Once the alleged extraocular muscle is found, it is not always easy to be sure it is truly muscle. Depending on the interval from the time the muscle was dislodged, fibrosis may have occurred. With fibrosis the structure loses the reddish striations characteristic of muscle, and it appears as a homogeneous combination of pink and tan, often blending into the surrounding tissues.

The oculocardiac reflex may be used at this time to the advantage of the surgeon. With the anaesthetist alerted, the surgeon applies traction to the suspected structure. If a significant bradycardia or dysrhythmia results, the surgeon can be sure the suspected structure is extraocular muscle, especially if it is reproducible. In a previous study of over 600 extraocular muscles $^{3}$ we found that $48 \%$ of the muscles receiving traction displayed a dysrhythmia or bradycardia exceeding $10 \%$. The medial rectus muscle displayed a positive response in $57 \%$ of cases. If traction of the suspected muscle results in no significant change in heart rate, however, the structure may still be an extraocular muscle that simply does not stimulate the vagal pathways significantly.

Before an operation for a slipped muscle the surgeon must instruct the anaesthetist to avoid the use of a parasympatholytic agent, such as atropine, given intravenously at the time of induction or during operation. Atropine and similar agents given intravenously inhibit or abolish the oculocardiac reflex, whereas these drugs given intramuscularly before operation do not have this effect.

To abolish the elicited reflex the surgeon and anaesthetist have a choice. If traction on the extraocular muscle is released, the heart beat usually reverts to normal. In the unusual circumstance of bradycardia or dysrhythmia continuing after traction is released, atropine given intravenously will generally restore the heart to a normal rhythm.

It is interesting that, despite the number of previous eye operations ( 6 in case 1, with 2 on the muscle that slipped), or the duration of time since the last eye operation on the dislodged muscle (6 years in case 2), one may still elicit the oculocardiac reflex.

\section{References}

${ }^{1}$ Bosomworth PP, Ziegler CH, Jacoby J. The oculo-cardiac reflex in eye muscle surgery. Anesthesiology 1958; 19: 7-10. ${ }^{2}$ Moonie GT, Rees DL, Elton D. The oculocardiac reflex during strabismus surgery. Can Anaesth Soc J 1964; 11: 621-632.

${ }^{3}$ Apt L, Isenberg S, Gaffney WL. The oculocardiac reflex in strabismus surgery. Am J Ophthalmol 1973; 76: 533-536.

${ }^{4}$ Rosenbaum AL, Metz HS. Diagnosis of lost or slipped muscles by saccadic velocity measurement. Am J Ophthalmol 1974; 77: 215-222.

${ }^{5}$ Scott AB. Active force tests in lateral rectus paralysis. Arch Ophthalmol 1971; 85: 397-404.

'Scott AB. Disinserted extraocular muscles. Am J Ophthalmol 1975; 79: 289-291.

'Parks MM. Ocular Motility and Strabismus. Hagerstown, Maryland: Harper and Row, 1975; 176. 